\title{
A 2D-DCT Image Processing in Matlab and Voice Informatics Based Remote Home Monitoring and Security System
}

\author{
Md. Biplob Hossain ${ }^{1.1}$ and M. Saifur Rahman ${ }^{2.1}$ \\ ${ }^{1}$ Department of Electrical and Electronic Engineering, \\ ${ }^{1}$ Rajshahi University of Engineering and Technology, Rajshahi-6204, Bangladesh. \\ biplobh.eee10@gmail.com ${ }^{1.1}$, msaifur.rahman.bd@ieee.org ${ }^{2.1}$
}

\begin{abstract}
This paper proposes, designs and constructs an image recognition \& voice technology based security system by highlighting the advantages of image processing technology and voice synthesis technology which are presence in the electronic market. This paper mainly approaches towards enhanced security by checking the tag image of the operator as well as recognizing voice which are validated previously by this system just using simply a web-camera or closed circuit television (CCTV) camera and a voice recording software system and gives the signal in terms of alarm, Alert Light, message via global system for mobile communication (GSM)/ general packet radio service (GPRS) to the consumer mobile number/the nearest police station's mobile number.
\end{abstract}

Keywords: Closed circuit television (CCTV), Light dependent resistor (LDR), Short message service (SMS), Synthesis, Two dimensional discrete cosine transform (2D-DCT), Two wire interface (TWI)

\section{Introduction}

An outlying home security, supervision and protection system have become more and more desirable with the progress of IT technology, network and automatic control technology now-a-days. Wireless sensor network (WSN) and global system for mobile communication (GSM) technology provide a better design and development of remote home security and supervene system whose main goal is to identify theft, fire etc., and send SMS to the respective house owner's mobile phone [1]. By visualizing home safety status on the controller desktop, laptop, PDA, mailing address or mobile phone, the user is always notifying with the current view of home. However, appropriate development of this method provides justification of the current safety level as well as to make summary judgment of home safety [2]. The need of security system is essential to make safety against crime and burglary acts that are the common issues now-a -days. Till now, a number of solutions on home security system are developed and some are implemented successfully in the market but most of these are either highly expensive or have lack of pleasant security. For example, "Wireless home network using 802.11 technology" in [3] paper suggests a wireless home network security known as Wi-Fi security system, which provides a medium for transferring media files. However, it is highly expensive and power consumer. "Security system against asset theft by using radio frequency Identification Technology" and "Positioning and tracking construction vehicles in highly dense urban areas and building construction sites" both papers highly represent, graphical user interface (GUI), is used in vehicle security system where the information is controlled via the GUI $[4,5]$. The system is activated when the tag image is read while the motor cycle is being located within the effective range. It's also highly expensive and power consumer. 
In this paper, we design a security system which gives remotely security against intrusion. Our work presents, the design and implementation of a smart security token system consisting of two technologies. We use web-cam or CCTV to capture images along a prescribed direction as first technology. The direction of capturing image will be controlled remotely using GSM/GPRS technology and send image equivalent digital signal to the house owner's mobile or mailing address. Voice synthesis is used as second technology in this system in order to record a voice and check the current record voice with the voices which are validated previously through this system.

\section{Proposed System Overview}

The proposed work consists of a microcontroller based electronic control device named processing section. It acts as project processor which takes input from image processing unit and sound recording unit through UART RS-232 protocol and provides output to $i$. the GSM module for messaging handler, ii. the door lock circuitry whether the door is opened or not iii. Light alarm circuitry whether it indicates "Green" or "Red". The overall system implementation probable view is given in Figure 1.

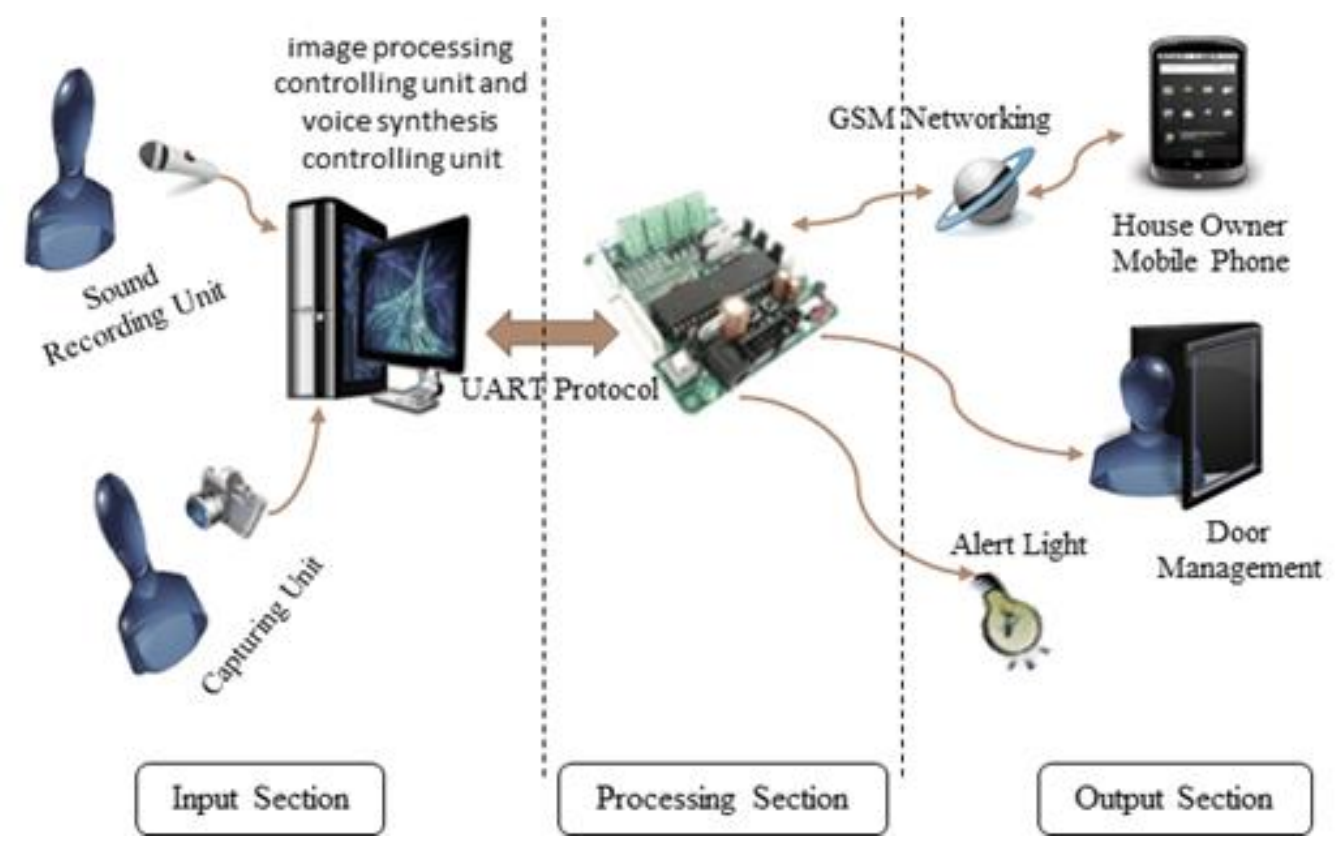

Figure 1. Overall Graphical View of Proposed Model. Input Section Reads Input from Capturing Unit and Voice Input Unit, Processing Unit Processes Input Information and Handover Result to the Output Section

The technical major complexity of the proposed security system is to merge computer, mobile \& microcontroller and synchronization among the three sections. The sequence of operation of the proposed system overly shown in the following functional block diagram. 


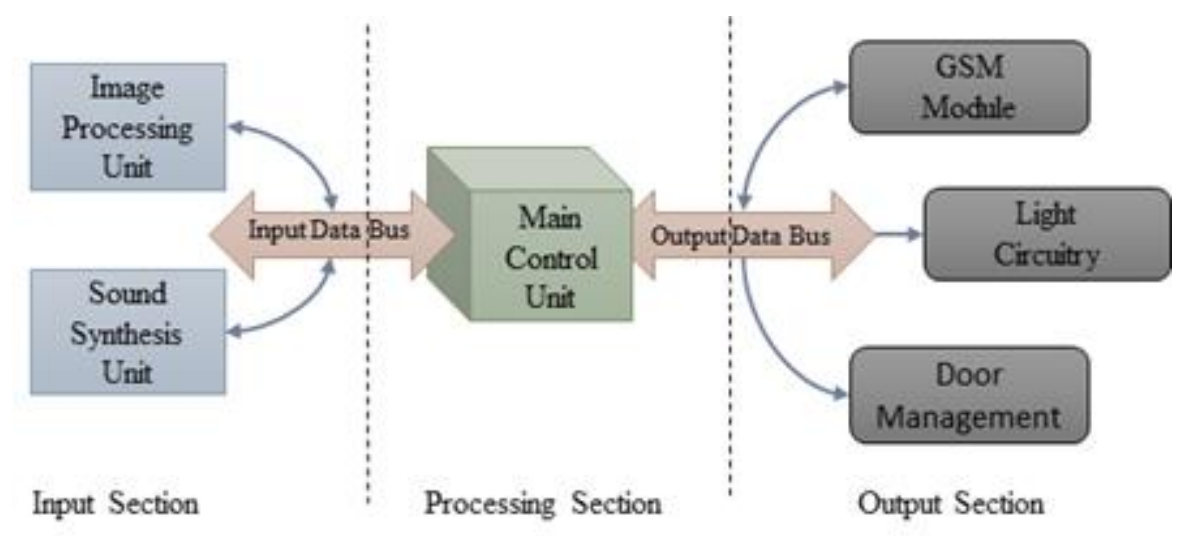

\section{Figure 2.Blockdiagram of Proposed System which Espress Data Flow Direction of Data Bus and the Natue of Data Bus}

Figure 2, indicates, input and output data bus which are bi-directional. In input section, RD/WR operations can be applied into both unit (image processing and sound synthesis), but in the output section, Light Circuitry and Door Management unit are capable of only $\mathrm{RD}$ operation that means data can only flow to light circuitry and door management unit. And GSM module unit is capable of both RD/WR operations. The algorithm of operation of the proposed security system is overly shown in the following flow chart inFigure 3.

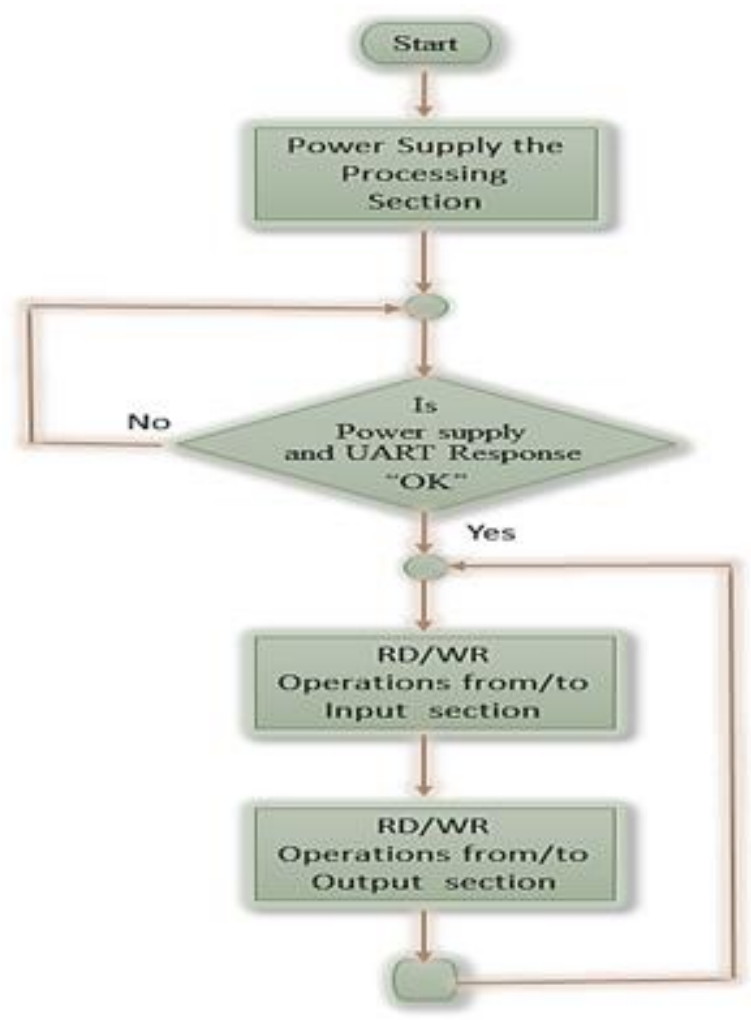

Figure 3.Flow Chart of Image Processing \& Voice Technology based Remote Detection and Security System 


\section{Proposed System Implementation}

In previous Overview passage, the overall system is classified into three sections, these are 1. Input Section, 2. Processing Section and 3. Output section.

\subsection{Input Section}

Input section consists of two unit, one is "Image processing technology" and another is "Voice synthesis technology".

3.1.1. Overview of Image Processing Technology: From some previous decades, image processing has been taken an advisable interest as bioinformatics research. A number of studies in previous on image processing and image computational technique have been done mentioned in [6-14]. In this paper, for building face recognition system in order to get proper security of the proposed model was done using the 2D-DCT algorithm. The proposed model has been effectively implemented by taking a great help from the works in [15-17]. In this project, "Matlab Image Acquisition Tool" and "Matlab Image Processing Tool" are used as image processing technology. "Matlab Image Processing Tool" has a verse application on the field of image processing. The front view of "Matlab Image Acquisition Toolbar" is given in Figure 4 at the moment of capturing an image to save it as authorized file for the system.

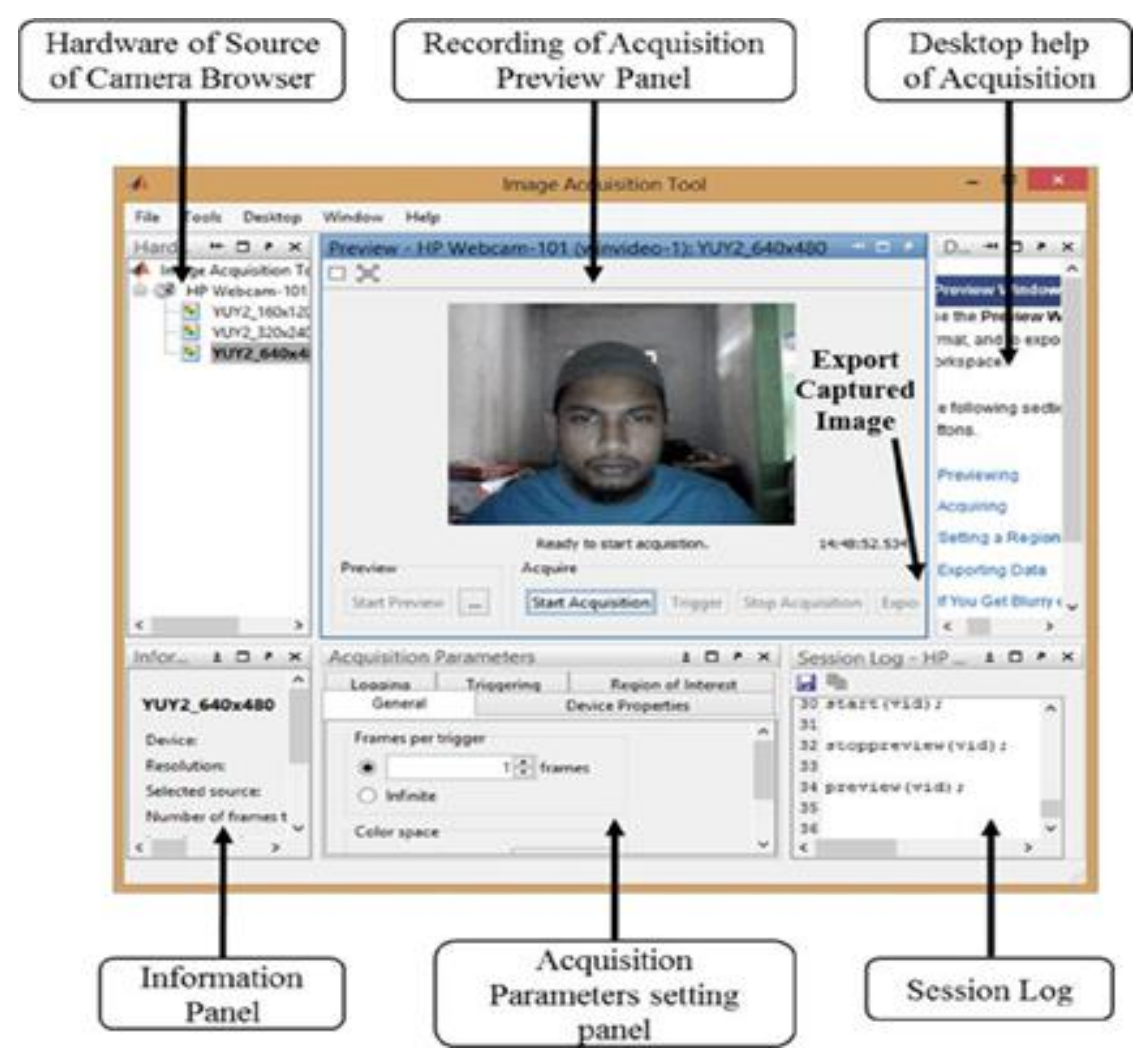

Figure 4.Font View of Image Acquisition Toolbox

Before starting acquisition of an image, we set acquisition parameters -data type as uint 8 bit and memory allocation to disk shown in Figure 5.aImage processing unit starts it action by acquitting an image and export it to MATLAB Workspace of a valid format similar of "file_name.mat". In Workspace exported file is automatically converted to uint 8, digital data with a specific bytes size is shown in Figure 5.b. 


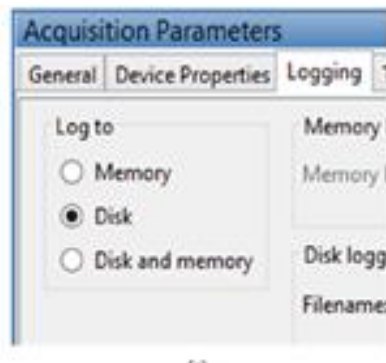

(2)

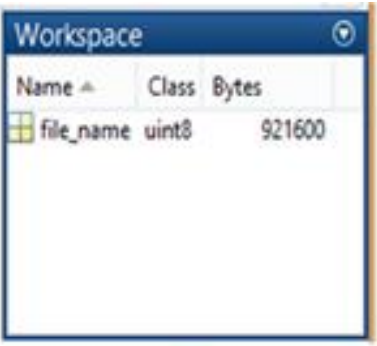

(b)

\section{Figure 5. Expressing (a) Memory Allocation to Disk where Processing Data} Would be Stored, (b) File to Workspace with Byte Size

Matlab is a regular environment for conducting experiments in the scope of image processing. One typical reason for containing a powerful Image Processing Toolbox. In particular, the matrix operations in Matlab are effective and efficient in the case of implementation of a lot of image processing algorithms [18]. Therefore, Matlab has been widely used to develop algorithms of image processing [19]. This paper presents a new algorithm for human face recognition. This algorithm uses the two-dimensional discrete cosine transform (2D-DCT). The DCT converts images from the spatial or time domain to the complex frequency domain. Since lower frequencies are more visually importance in an image than higher frequencies, the DCT reveals high-frequency coefficients and quantizes the remaining coefficients. This procedure suppresses data volume without losing too much image quality [20]. The 2D-DCT of an $\mathrm{M} \times \mathrm{N}$ matrix $\mathrm{A}$ is defined-asfollows:

$B_{p q}=\alpha_{p} \alpha_{q} \sum_{m=0}^{M-1} \sum_{n=0}^{N-1} \mathrm{~A}_{\mathrm{M}}\left(\cos \frac{\Pi(2 m+1) p}{2 M}\right) *\left(\cos \frac{\Pi(2 n+1) q}{2 N}\right), 0 \leq p \leq M-1,0 \leq q \leq N-1 \ldots \ldots \ldots \ldots(1)$

The values $B_{p q}$ is the DCT coefficients. The DCT is an invertible transform, and the 2D-IDCT (2D Inverse-DCT) is defined as follows:

$$
A_{m n}=\alpha_{p} \alpha_{q} \sum_{p=0}^{M-1} \sum_{q=0}^{N-1} B_{p q}\left(\cos \frac{\Pi(2 m+1) p}{2 M}\right) *\left(\cos \frac{\Pi(2 n+1) q}{2 N}\right), 0 \leq m \leq M-1,0 \leq n \leq N-1 \ldots \ldots \ldots . \text {. (2) }
$$

Face identification digitally has become a great active area of research in recent years mainly due to increase security demands and its potentiality in commercial purpose and high demandable applications [18].

Face image fabrication process of different accessible user are taken with "Matlab Image Acquisition Tool" discussed in earlier is shown in Figure 6. Image pre-processing includes the following steps [21],

- Auto adjusting hue and saturation levels

- Adjusting brightness and contrast to fixed scale

- Desaturation of 24 bit RGB color into 8 bit grayscale

- Downsizing images to $512 \times 512$ pixels

- Saving images in jpg form. 


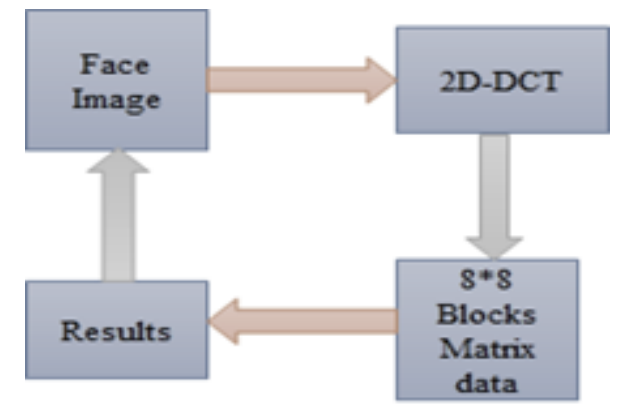

Figure 6. Proposed Technique for Face Recognition System

If any image data available in the Workspace then this file is imported to Matlab Simulink windows using "From Workspace" block. And display the data value on "Display Sink" block and finally send it to microcontroller based processing unit via "RS232 COM-13" port using microcontroller featured "UART". The "Matlab Simulink tool" using in image processing technique is visualized in Figure 7.

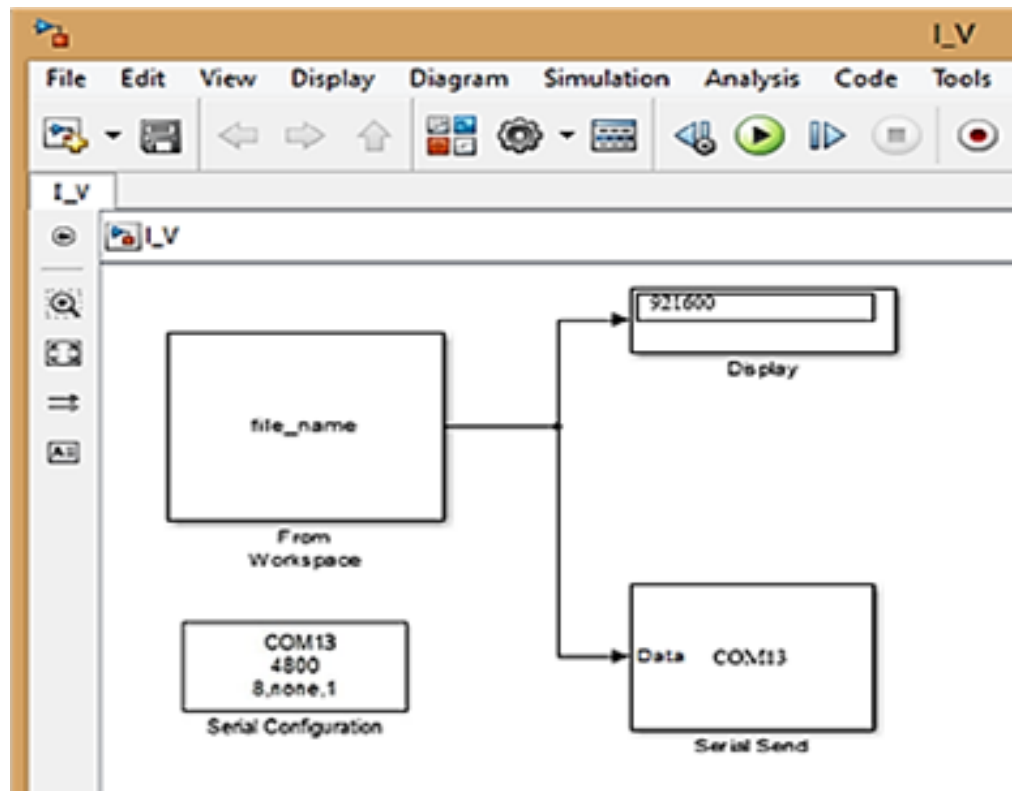

Figure 7.Control Representation of Image Processing Technique Telling the Readable Data which is Available in COM-13 Port. It is Displayed at Display Sink on Matlab

The data available in COM-13 Buffer is received as input by the microcontroller based processing unit. Then processing unit tries to match it in size with the validated data which is already registered in this system. So finally it has been concluded thefunction of image processing technique is a sequentially complex. The layout, used for image processing in this system as well as algorithm of image modeling are given in Figure 8. 


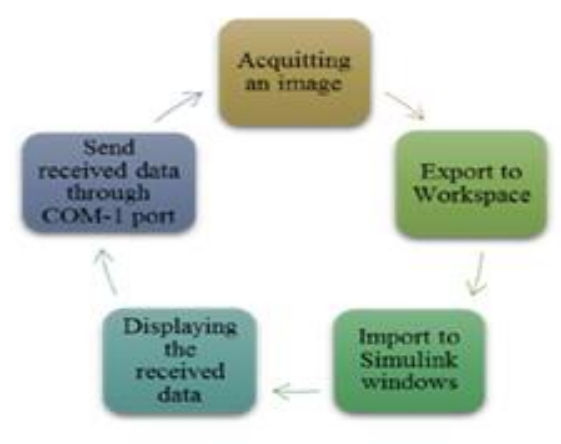

(a)

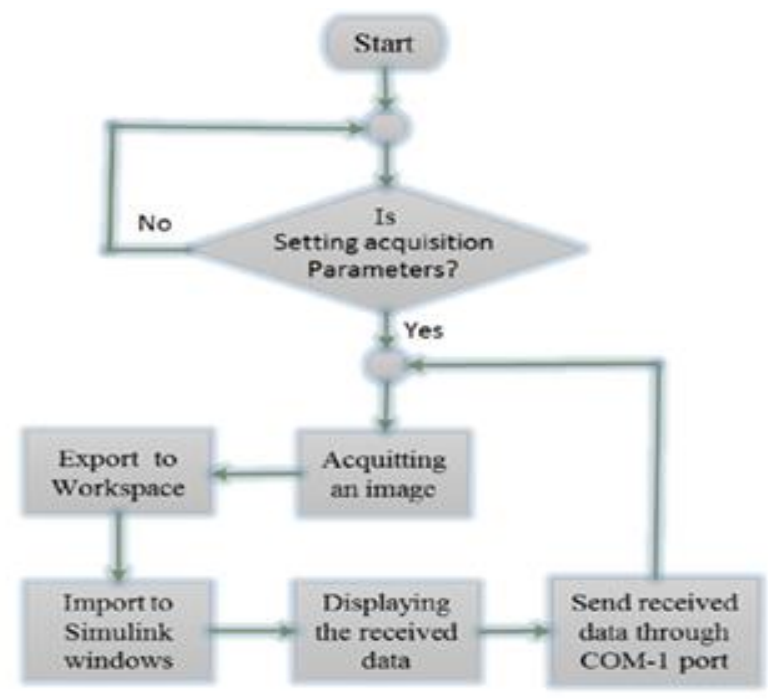

(b)

\section{Figure 8. (a) Cyclic Layout and (b) Flow Chart of Image Processing Unit}

3.1.2. Overview of Voice Synthesis Technology: In this project, a software for Windows-8 is developed named "Voice Syntheser for Security" to take voice command from a sound source such as human voice through headphone. This software is developed using C\# object oriented programming. The front view of "Voice Syntheser for Security" software while a COM port connection is available, is shown in Figure 9.

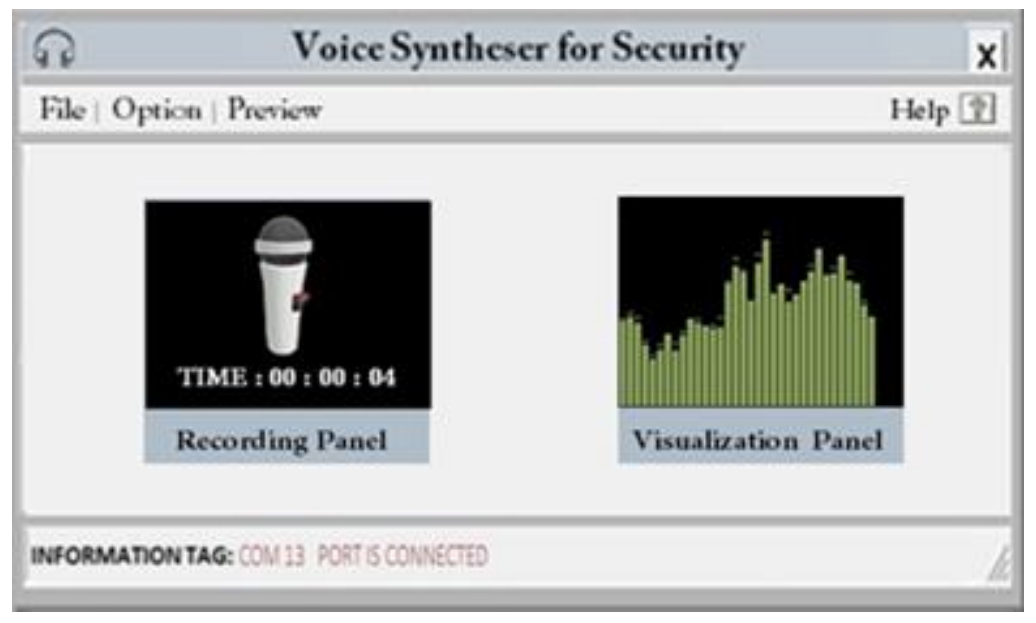

Figure 9.Front view of Voice Syntheser for Security

The main task of "Voice Syntheser for Security" is to take a sound for a time interval of 5 seconds (time interval is user changeable) via headphone and after that interval, it converts receiving sound to file_name.wav format with a specific byte size and generates a single digit automatically according to the file byte size value and finally sends single digit value to the connected COM port. The operational flow chart of voice synthesis technology is shown in Figure 10. 


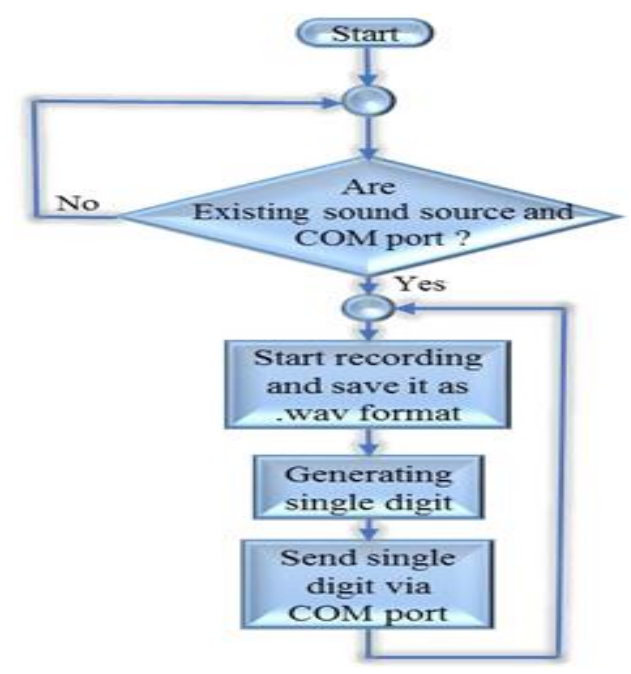

\section{Figure 10.Flow Chart of Voice Synthesis Technology. The Process of Recording of Voice is Started when Only a Com Port is Plugged in into the Computer}

\subsection{Processing Section}

Processing unit consists of a microcontroller named "Atmega 32", works simply as project brain. Processing unit takes input from input section, processes and validates it and finally provides response to the output section according to the input. The logical arrangement and algorithm of operation of processing section is given in Figure 11.

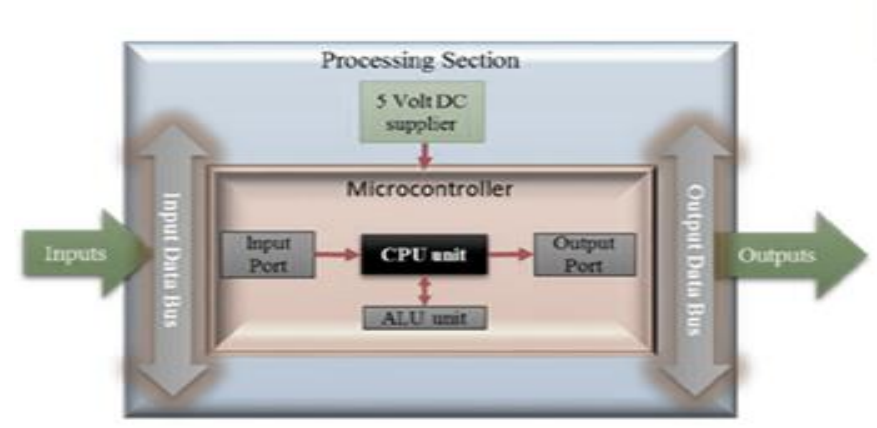

(a)

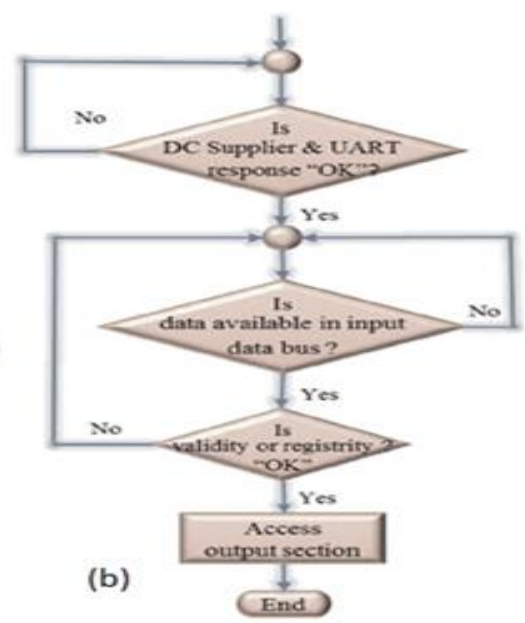

Figure 11. (a) Logical Arrangement, (b) Algorithm of Operation of Processing Section

\subsection{Output Section}

Output section consists of a couple of LEDs, one is "Red" and the rest is "Green" work as alert light, door management system and GSM communication system. If validated or registered input signal is received then a "user access message" is sent to the house owner mobile number through GSM module "SIM-300". Alert light indicates "Green" and door is opened and if invalidated or unregistered input signal is received then an "intrusion alert message" is sent to the house owner mobile number then alert light indicates "Red" and door will never be opened. 


\section{Project Model Communication Protocol}

The major difficulty of communication of the proposed security system is to communicate a single microcontroller with GSM module and with computer at a time. To make communication process easier among computer, microcontroller and GSM module, two programming features of microcontroller are needed to use, one is conventional "Universal Asynchronous Receiver Transmitter familiarly known "UART programming" and another is Two Wire Serial Interface programming or "TWI" programming. UART programming is used for communicating microcontroller with computer and TWI programming is used for communicating with GSM module.

The Two-wire Serial Interface (TWI) is ideally suited for commercial microcontroller applications [22] in which up to 128 different devices are made interconnection [22]. In this project, one slave device "SIM-300" GSM module is connected to SDA and SCL line as like as following Figure 12.

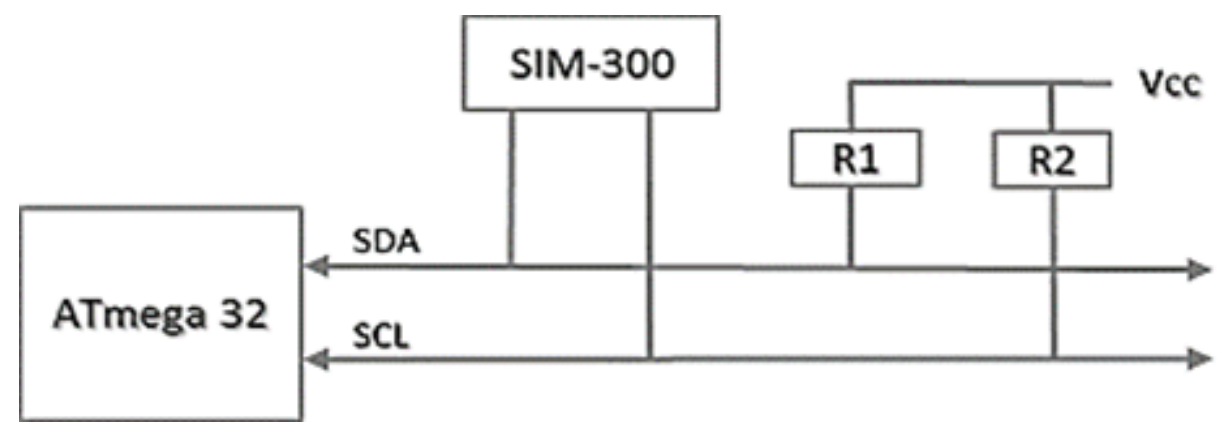

Figure 12. TWI Bus Interconnection for Proposed Model

The overall circuit diagram of processing section is shown in Figure 13.

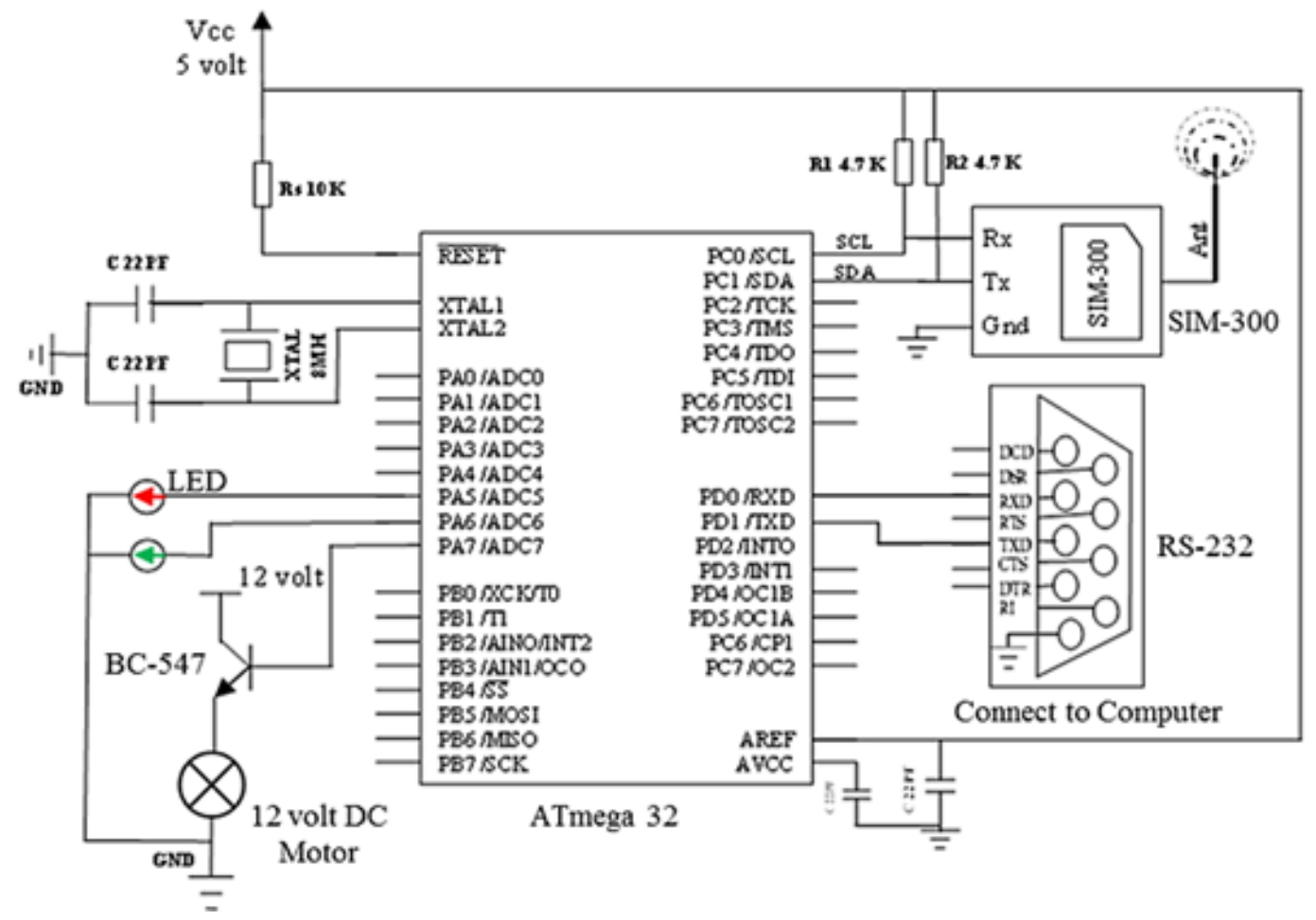

Figure 13. The Overall Circuit Diagram of Processing Section 


\section{Proposed Model Software Simulation and Results}

The analytical device operation is simulated using Proteus 7 professional software. In Proteus 7 simulation section, communication between microcontroller and SIM-300 is absent due to unavailability SIM-300 component in the software library. The simulated circuit diagram, designed in Proteus software just before the moment of reading an input from input section (image processing unit \& sound synthesis unit) is shown in Figure 14.

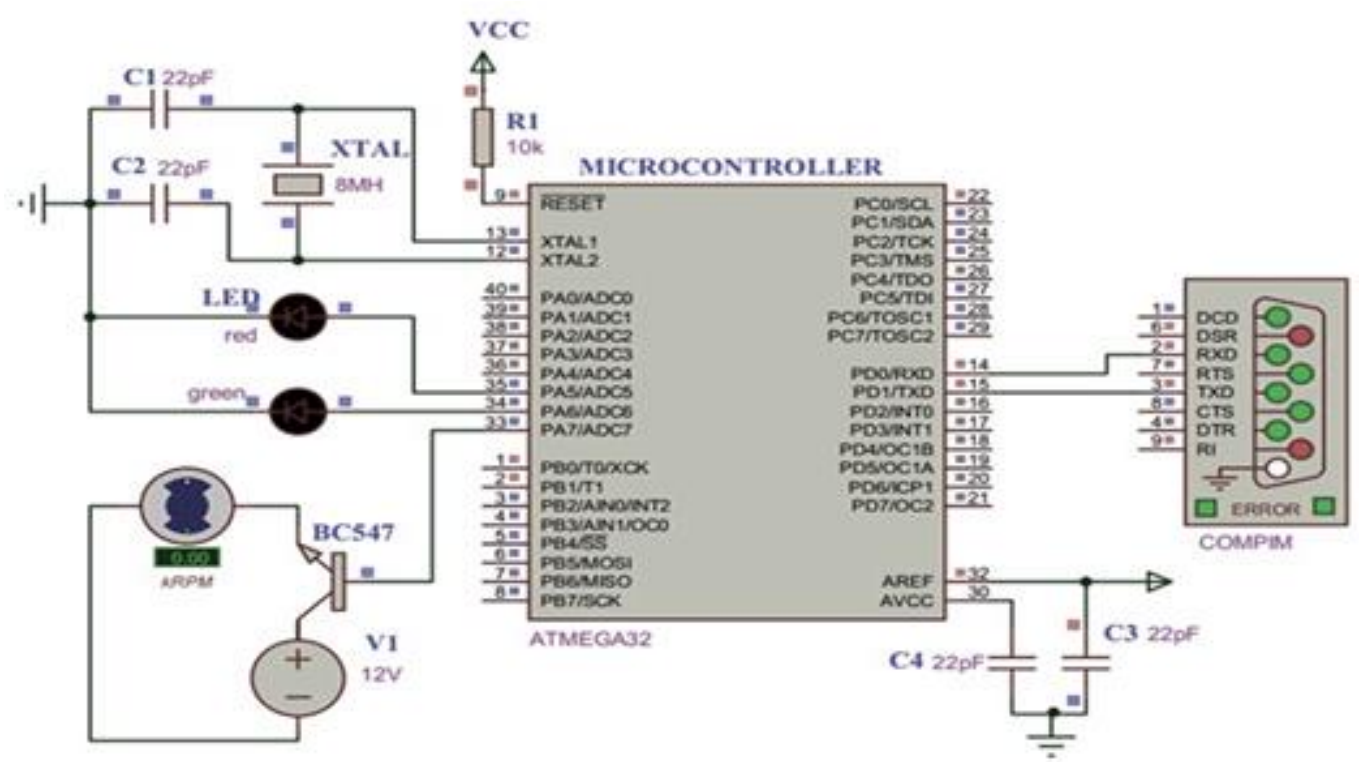

Figure 14. The Simulated Circuit Diagram for Processing Section

A snapshot is shown in Figure15 which indicates, motor is off mode (inactive door management system), Red LED is on mode and send an alert message "An invalid user tried to access the security system" to the house owner mobile no. that is absence in Proteus simulation when an unauthorized or unregistered input is received both from image processing unit and voice synthesis unit.

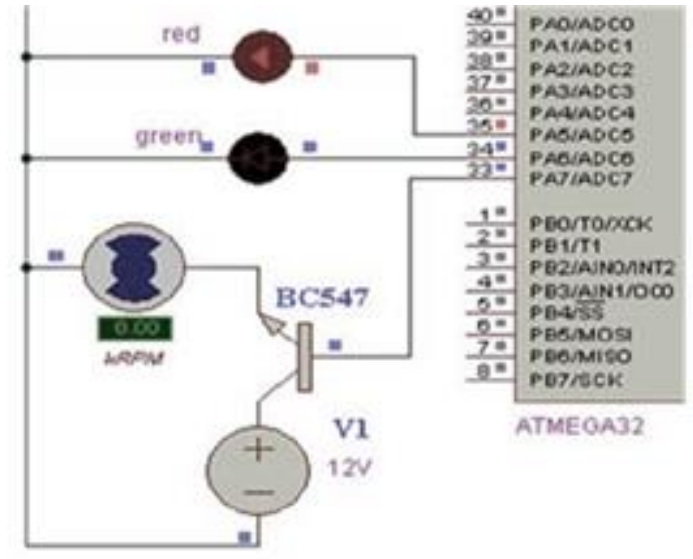

(a)

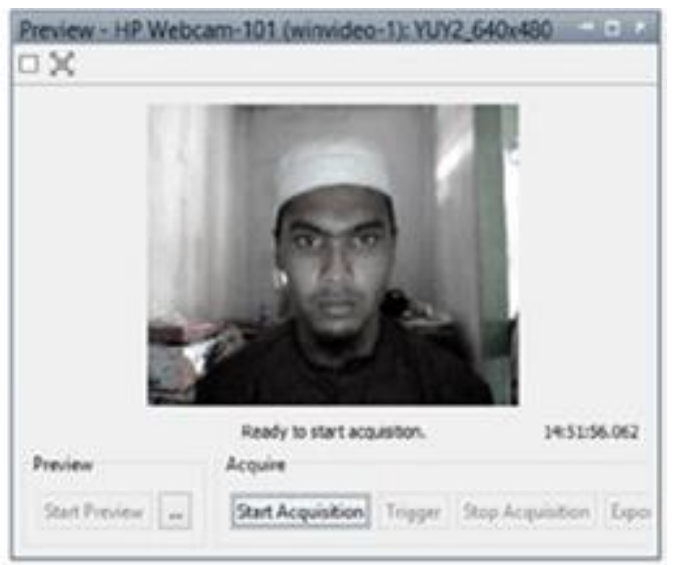

(b)

Figure 15. (b) Invalid image Access and (a) Observation of Output in Terms of LED, Exhaust Fan 
Figure 16 is the representation of the $\mathrm{ON}$ mode device when an authorized or registered input is received both from image processing unit and voice synthesis unit.

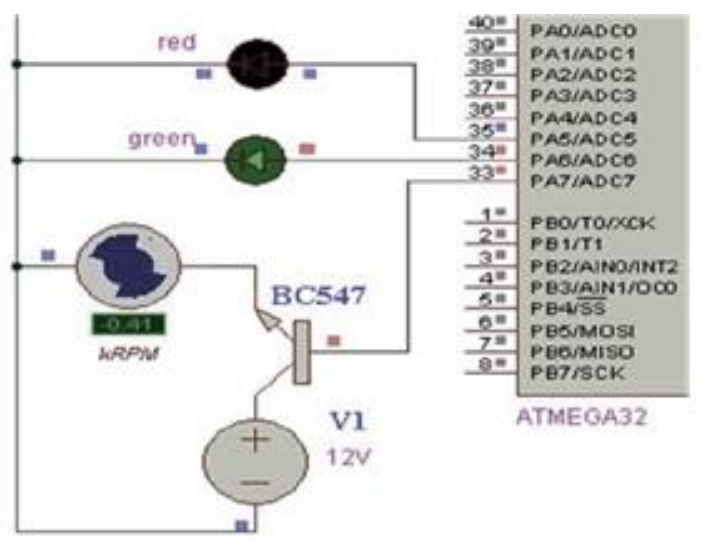

(a)

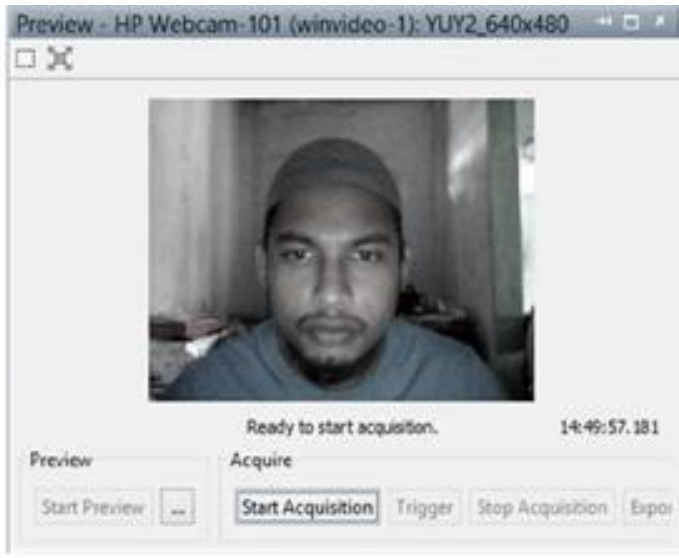

(b)

Figure 16. Response of Processing Section when Authorized Image is Captured

\section{Conclusions and Future Improvement}

The system described in this paper offers the best performance. It ensures the most valuable detection of any type of intrusions and can take necessary actions. It is an efficient and cost-effective system and the output is more acceptable as an accurate which is implemented as prototype using MATLAB and practically examined. The next version of this device will be demonstrated soon with a basic development. Firstly, it will be upgraded by using GPRS and sending data to the consumers through E-mail and sends continuous alert message to the nearest Police Station if the intrusion is occurred.

\section{Acknowledgement}

This work is supported in whole by Funds for publication from the SERSC Korea branch. We would greatly thankful to Dr. Md. Masud Rana for his guidance and deliverance of many valuable advices throughout a long time of our project study.

\section{References}

[1] A. Aggarwal and R. C. Joshi, "WSN and GSM based Remote Home Security System", Int. Conf. on Recent Advances and Future Trends in Information Technology (iRAFIT), Proceedings published in International Journal of Computer Applications(IJCA), (2012).

[2] N. Subramanian, "Visualizing safety score for the digital home", Nicole, (2008), pp. 128-134.

[3] L. Ophir, "802.11 over coax-a hybrid coax -wireless home network using 802.11 technology", Proc. Consumer Communications and Networking Conference, (2004).

[4] N. Jinaporn, S. Wisadsud, P. Nakonrat and A.Suriya, "Security system against asset theft by using Radio frequency Identification Technology", Proc. ECTI -CON, (2008).

[5] M. Lu, W. Chen, X. Chen, H.C. Lam and J. Liu, "Positioning and tracking construction vehicles in highly dense urban areas and building construction sites", Automation in Construction, vol. 16, (2007), pp. 647-656.

[6] J. M. Adant, "Block operations in digital signal processing withapplications to TV coding", Signal Process., vol. 13, (1987), pp. 285-397.

[7] K. N. Ngan, "Experiments on 2D decimation in time and orthogonal transform domains", Signal Process., vol. 11, (1986), pp. 249-263. 
[8] B. Natarajan and B. Vasudev, "A fast approximate algorithm for scaling down digital images in the DCT domain”, Proc. IEEE Int. Conf. on Image Processing, (1995); Washington, DC.

[9] S. Martucci, "Image resizing in the DCT domain", Proc. IEEE Int. Conf. on Image Processing, (1995); Washington, DC.

[10] H. T. Fung and K. J. Parker, "Segmentation of scanned documents for efficient compression", Proc. SPIE: Visual Communications and Image Processing, (1996); Orlando, FL.

[11] S. N. Srihari, "Document image understanding", Proc. Int. Symp.Circuits and Systems, (1986).

[12] P. Biber, S. Fleck and W. Straber, "A probabilistic framework for robust and accurate matching of point clouds", $26^{\text {th }}$ Pattern Recognition Symposium (DAGM), (2004).

[13] A. Jepson and M. Black, "Mixture models for optical flow computation", IEEE Conference on Computer Vision and Pattern Recognition, (1993).

[14] H. Hachama, F. Richard and A. Desolneux, "A mammogram registration technique dealing with outliers", Proc. of the IEEE International Symposium on Biomedial Imaging (ISBI'), (2006).

[15] W. B. Pennebaker and J. L. Mitchell, “JPEG: Still Image Compression Standard”, (1993).

[16] K. R. Rao and P. Yip, "Discrete Cosine Transform: Algorithms, Advantages, Applications", (1990); New York.

[17] Ricardo L. de Queiroz, "Processing JPEG-Compressed Images and Documents", IEEE Transactions on Image Processing, vol. 7, no. 12, (1998), pp. 1661-1672.

[18] A. Abdallah, M. Abou El-Nasr and A. Lynn Abbott, "A new face detection technique using 2d dct and self organizing feature map", Proc. of World Academy of Science, Engineering and Technology, (2007).

[19] R. F. Frangi, W. J. Niessen, K. L. Vincken and M. A. Viergever, "Multiscale vessel enhancement filtering", (1998),pp.130-137.

[20] J. Nagi, "Design of an Efficient High-speed Face Recognition System", Department of Electrical and Electronics Engineering, College of Engineering, University Tenaga National, (2007).

[21] Jawad Nagi, Syed Khaleel Ahmed and Farrukh Nagi, "A matlab based face recognition system using image processing and neural networks", 4th International Colloquium on Signal Processing and its Applications, , Faculty of Electrical Engineering, (2008); Kuala Lumpur, Malaysia.

[22] Atmel Microcontroller User Manual, Motorola Products Corporation, http://www.atmel.com.

\section{Authors}

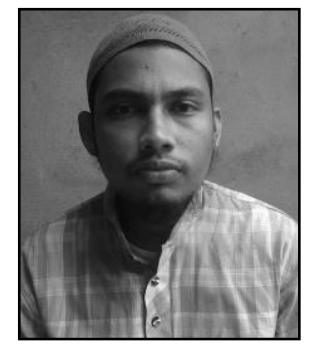

Md. Biplob Hossain (S' 2014) is with department of Electrical and Electronic Engineering, Rajshahi University of Engineering and Technology, Rajshahi-6204, Bangladesh. Currently he isthe chiefcourse coordinator at Point Tech Bd., an engineering firm at Dhaka,Bangladesh (www.pointtechbd.in). His research interests arecomputational electromagnetic, E-M fields, microwave theory and devices, graphene modeling and applications, image processing, signal processing, bio-medical and bio-informatics engineering.

E-mail: biplobh.eee10@gmail.com, mohammad.hossain.biplob@gmail.com

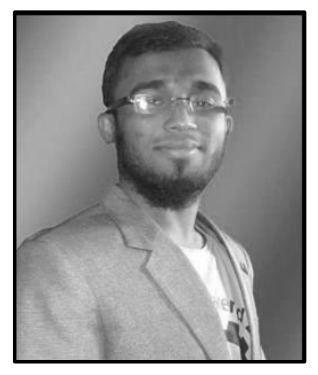

M. Saifur Rahman(S' 2014)currently works as student of Electrical and Electronic Engineering, Rajshahi University of Engineering and Technology, Rajshahi-6204, Bangladesh, His research interests included bioinformatics and biomedical engineering, signal processing, image processing, and automotive System. He is currently IEEE student member.

E-mail: msaifur.rahman.bd@ieee.org, saifurrahman121042@gmail.com 